\title{
Síntese de um Novo Derivado de Sulfonamida a Partir da Cumarina-6-Clorossulfonada
}

\author{
Synthesis of new Sulfonamide Derivated from Coumarin-6-Sulfonyl Chloride
}

\author{
Adélia Segin Vale Velosa ${ }^{\mathrm{a}}$; Vagner Pereira da Silva ${ }^{\mathrm{b}}$; Susana Nogueira Diniz ${ }^{\mathrm{ac}}$; Eunice Okuyama ${ }^{\mathrm{b}}$; \\ Márcio Luiz dos Santos ${ }^{\mathrm{ac}}$; Regina Mara Silva Pereira ${ }^{\text {ac }}$ \\ ${ }^{a}$ Universidade Anhanguera de São Paulo, Programa de Pós-Graduação Stricto Sensu em Biotecnologia e Inovação e Saúde. SP, Brasil.

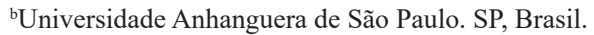 \\ 'Universidade Anhanguera de São Paulo, Programa de Pós-Graduação Stricto Sensu em Farmácia. SP, Brasil. \\ *E-mail:
}

\begin{abstract}
Resumo
Sulfonamida é uma classe de compostos de interesse para obtenção de novos medicamentos por apresentar propriedades químicas e biológicas como antitumoral, antimicrobiana, antifúngica, anti-inflamatória, antiobesidade, diurético, hipoglicêmico, anti-neuropática, entre outras. Mais de trinta medicamentos contendo esse grupo funcional estão em uso clínico, incluindo antibacterianos, diuréticos, anticonvulsivantes, hipoglicêmicos e inibidores de protease do vírus da imunodeficiência humana (HIV). Aproximadamente, 150 diferentes sulfonamidas são comercializadas como agentes bactericidas, contendo variações estruturais para potencializar a eficiência e solubilidade em sistemas biológicos. É importante estabelecer métodos rápidos e simples para a síntese de novas sulfonamidas com rendimento satisfatório. Nesse trabalho foi relatado um método sintético eficiente e fácil para obter sulfonamidas à partir da cumarina-6-clorossulfonada e as aminas primárias, tercbutilamina (CSNtert), isobutilamina (CSNiso) e isopropilamina (CSNisop) com rendimento de 71\%. Essas novas sulfonamidas à base de cumarina foram caracterizadas por análise elementar, espectroscopia ultravioleta, espectroscopia no infravermelho por refletância total atenuada (ATR) e Ressonância Magnética Nuclear de ${ }^{1} \mathrm{H} \mathrm{e}{ }^{13} \mathrm{C}$. Além disso, foram realizados testes de condutividade e ponto de fusão. A caracterização espectroscópica e química confirmou a formação de novos compostos e foi possível propor sua estrutura. Estudos da citotoxicidade da CSNtert, CSNiso e CSNisop em células epiteliais normais mostrou que estes compostos apresentam menor citotoxicidade que a cumarina-6-clorossulfonada.
\end{abstract}

Palavras-chave: Cumarina. Sulfonamida. Síntese

\section{Abstract}

Sulfonamide is an important class of compounds that play an important role in the discovery of new medicines due to its broad chemical and biological activities such as antitumoral, antimicrobial, antifungic, anti-inflammatory, anti-obesity, diuretic, hypoglycemic, anti-neuropathic pain activities, among others. More than 30 drugs containing this functional group are in clinical use, including, antibacterial, diuretics, anticonvulsants, hypoglycemic and human immunodeficient virus (HIV) protease inhibitors. Approximately 150 different sulfonamides have been sold as bactericidal agents, and structural variations seems to increase their efficiency, potency and solubility in biological systems. Fast and simple synthetic methods for the construction of new sulfonamides with satisfactory yielding are important to be stablished. In this work an efficient and easy synthetic method was reported to obtain sulfonamides from coumarin-6-sulfonyl chloride and primary amines, tert-butyl amine (CSNtert), isobutylamine (CSNiso) and isopropylamine (CSNisop) with excellent yield, (71\%). These new coumarin-based sulfonamides were characterized by elementary analysis, spectroscopy UV, Infrared by Attenuated Total Reflectance (ATR) and Nuclear Magnetic Resonance of ${ }^{1} \mathrm{H}$ and ${ }^{13} \mathrm{C}$. In addition, conductivity tests were performed and melting point determined. The spectroscopic and chemical characterization confirmed the structures of the new compounds and could be possible to propose the structure. Studying their cytotoxicity against normal epithelial cells shows that the new compounds have lower cytotoxicity than coumarin-6-sulfonyl chloride.

Keywords: Coumarin. Sulfonamide. Synthesis

\section{Introdução}

Acumarina é um heterociclo contendo oxigênio encontrada em plantas como canela, guaco, lavanda, hortelã-pimenta e apresenta diversas propriedades biológicas (KUMAR et al., 2015; NAJMANOVA et al., 2015; LUNAZZI et al., 2010). A cumarina e seus derivados têm sido usados como precursores para a síntese de uma variedade de agentes medicinais (CUNHA et al., 2019) e intermediários na química orgânica sintética (GÖLCÜ, 2006). Novos derivados, ainda estão sendo descobertos e sintetizados devido as potencialidades apresentadas.

Compostos a base de sulfonamidas são uma importante classe de moléculas que apresentam estruturas químicas de interesse e potencial biológico para uso na medicina (SINGH; RAJOUR.; PRAKASH, 2012; NOFAL; EL-ZAHAR; ABD EL-KARIM, 2000). Há décadas compostos contendo grupamento sulfomamida são usados como antimicrobiano (ZANI; VICINI, 1998). São relatadas ainda ação antitumoral, antifúngica, antituberculosa, anti-inflamatória (atividade inibitória da anidrase carbônica e COX-2). (KURT et al., 2019; NAM; KIM, 2015; LIU et al., 2012; LEE et al., 2013; NEWMAN; CRAGG, 2012; BRYDA et al., 2019; GROVER; JACHAK, 2015; SABT et al., 2018; PINTO; SILVA, 2017). Devido às propriedades biológicas e atividades 
farmacológicas de compostos à base de sulfonamida e cumarinas nesse trabalho decidiu-se sintetizar sulfonamidas a partir da cumarina-6-clorossulfonada e aminas primárias como precursores.

Nesse trabalho, a cumarina-6-clorossulfonada foi preparada a partir da sulfonação da cumarina, seguido do acoplamento das aminas primárias, tercbutilamina, isobutilamina e isopropilamina. As sulfonamidas sintetizadas foram caracterizadas por análise elementar, espectroscopia UV-VIS, espectroscopia no infravermelho por refletância total atenuada (ATR) e ${ }^{1} \mathrm{He} \mathrm{e}^{13} \mathrm{C} \mathrm{NMR}$. Além disso, foram realizados testes de condutividade, ponto de fusão e citotoxicidade em células epiteliais normais. A partir dessas análises foi possível propor à estrutura dos novos compostos.

\section{Material e Métodos}

Os espectros de ressonância magnética nuclear de próton e carbono, RMN de ${ }^{1} \mathrm{H} \mathrm{e}{ }^{13} \mathrm{C}$, foram obtidos no espectrômetro Bruker Avance III, operando a 300/75 MHz. Clorofórmio deuterado e acetona deuterada, $\mathrm{CDCl}_{3}$ e $\mathrm{CD}_{3} \mathrm{OD}$ (Tedia Brazil) foram utilizados como solvente e o pico residual de solvente não deuterado como padrão interno. Os desvios químicos $(\delta)$ foram relatados em ppm e a constante de acoplamento $(J)$ em Hz. Os espectros no infravermelho (IV) foram obtidos em estado sólido por refletância, entre 400 e $4000 \mathrm{~cm}^{-1} \mathrm{em}$ um espectrofotômetro Nicolet-Nexus. O ponto de fusão foi medido usando o equipamento QUIMIS, e os espectros ultravioleta (UV) foram obtidos em uma solução de metanol entre 200 e $500 \mathrm{~nm}$ em um espectrômetro Femto 800-XI. Todos os produtos químicos utilizados cumarina, ácido clorossulfônico, isobutilamina, tercbutilamina e dietilamina foram obtidos da Sigma-Aldrich e Merck. Todos os solventes usados foram grau PA.

\subsection{Síntese da cumarina-6-clorossulfonada (CCIS)}

A reação de sulfonação foi realizada de acordo com Becker (BECKER; BERGER, 2009) para inserção do grupo sulfônico em compostos heterocíclicos. Essa metodologia foi utilizada com modificações para a clorossulfonação da cumarina (1,2-benzopirona). $13.7 \mathrm{mmol}$ de cumarina foi solubilizada em $5 \mathrm{~mL}$ de clorofórmio e $2.73 \mathrm{mmol}$ de ácido clorossulfônico foi adicionado. A reação foi mantida sob refluxo e agitação por 4 horas. Após esse período, o sistema foi resfriado em banho de gelo e adicionada água destilada à mistura para precipitação da cumarina-6-clorossulfonada (CClS). A CCIS foi filtrada, purificada por recristalização em água e seca a $40^{\circ} \mathrm{C}$. O composto foi identificado por ponto de fusão, espectroscopia no ultravioleta, infravermelho, RMN de ${ }^{1} \mathrm{H} \mathrm{e}{ }^{13} \mathrm{C}$, análise elementar e condutividade.

\subsection{Síntese geral das sulfonamidas}

As sínteses das novas sulfonamidas foram realizadas a partir da inserção das aminas (tercbutilamina, dietilamina ou isopropilamina) no grupo da cumarina-6-clorossulfonada (CCIS) (VALDUGA; STEFANI; PETRAGNANI, 2001).

Em um balão de $50 \mathrm{~mL}, 1.0 \mathrm{mmol}$ de cumarina-6clorossulfonada (CClS) foi solubilizada em $30 \mathrm{~mL}$ de acetona e aquecida até $90^{\circ} \mathrm{C}$. Depois de misturar $1 \mathrm{mmol}$ de carbonato de sódio e $1 \mathrm{mmol}$ de amina em $10 \mathrm{~mL}$ de água, foi adicionada à solução da CCIS e a mistura foi mantida sob refluxo por $3 \mathrm{~h}$.

Após esse período, a solução foi resfriada e foram adicionados $5 \mathrm{~mL}$ de clorofórmio e $5 \mathrm{~mL}$ de água. A fase orgânica foi separada da aquosa, lavada três vezes e adicionado $\mathrm{MgSO}_{4}$ (sulfato de magnésio) para remover a água residual. O $\mathrm{MgSO}_{4}$ foi removido por filtração e o solvente orgânico foi removido por vácuo.

\subsection{Ensaio de viabilidade celular}

A linha celular renal epitelial normal (VERO - code: 0245), da coleção de células do Rio de Janeiro (BCRJ) foi cultivada em meio RPMI 1640 suplementado com bicabonato de sódio 2g/L ("LCG Biotecnologia"), estreptomicina 100 $\mu \mathrm{g}$ / $\mathrm{mL}$, penicilina $100 \mathrm{UI} / \mathrm{mL}$ e $10 \%$ de soro bovino fetal. As células foram mantidas a $37^{\circ} \mathrm{C}$ e $5 \%$ de $\mathrm{CO}_{2}$. Para medir a viabilidade celular foi utilizado o MTT (brometo de tiazolil azul de tetrazólio - Sigma-Aldrich). As células foram semeadas em placas de 96 poços à densidade de $1 \times 10^{4}$ células/ poço e incubadas $\left(37^{\circ} \mathrm{C}, 5 \% \mathrm{CO}_{2}\right)$. Em seguida, os compostos dissolvidos em dimetilsulfoxido (DMSO) foram adicionados e as placas foram incubadas por 24 horas. A concentração final da CClS, CSNtert, CSNiso and CSNisop foi de 350 a 2800 $\mu \mathrm{M}$. Após 24 horas, o meio foi substituído por um meio fresco $(200 \mu \mathrm{L})$ contendo MTT $(0.5 \mathrm{mg} / \mathrm{mL})$. As células foram então encubadas por 4 horas, o sobrenadante foi coletado, DMSO $(200 \mu \mathrm{L})$ foi adicionado, agitando por $5 \mathrm{~min}$ até os cristais estarem completamente dissolvidos. A absorbância foi medida a $540 \mathrm{~nm}$ usando um leitor de microplantas (Labtech LT-4000 MS) e correlacionada diretamente com o número de células viáveis na cultura. Os resultados foram expressos em IC50 (Concentração que mata $50 \%$ das células) e todos os testes foram feitos em triplicatas.

\section{Resultados e Discussão}

A cumarina-6-clorossulfonada (CClS) foi sintetizada por reação de substituição eletrofílica do $\mathrm{ClSO}_{2} \mathrm{OH}$ no anel aromático da cumarina de acordo com o método de Becker (Figura 1) (BECKER; BERGER, 2009). O composto CCIS foi caracterizado por $\mathrm{UV}$, IR, $\mathrm{RMN}{ }^{1} \mathrm{H}$, análise elementar, condutividade e ponto de fusão. Nos espectros no IV foram observadas duas bandas de 1373 e $1167 \mathrm{~cm}^{-1}$, característica de $v \mathrm{~S}=\mathrm{O}$ (grupo sulfonila), e bandas de $\mathrm{C}=\mathrm{C}$ e $\mathrm{C}=\mathrm{O}$ foram deslocadas para uma frequência mais alta quando comparadas à cumarina devido à substituição de um $\mathrm{H}$ aromático pelo grupo $\mathrm{SO}_{2} \mathrm{Cl}$ (Quadro 1). 
Quadro 1 - Condutividade molar $\left(\mathrm{ohm}^{-1} \cdot \mathrm{cm}^{2} \cdot \mathrm{mol}^{-1}\right)$ a $25^{\circ} \mathrm{C}$, análises elementares e ponto de fusão $\left({ }^{\circ} \mathrm{C}\right)$ da CCIS, CSNiso, CSNtert e CSNisop

\begin{tabular}{|c|c|c|c|c|c|}
\hline \multirow{2}{*}{ Compostos } & \multirow{2}{*}{ Condutividade } & $\begin{array}{c}\text { \% C } \\
\text { Exp (Calc) }\end{array}$ & $\begin{array}{c}\text { \% H } \\
\text { Exp (Calc) }\end{array}$ & $\begin{array}{c}\text { \% N } \\
\text { Exp (Calc) }\end{array}$ & Ponto de Fusão \\
\hline CClS & 156.2 & $44.18(44.00)$ & $1.90(2.05)$ & - & $110-112$ \\
\hline CSNIso & 29.3 & $55.30(55.50)$ & $5.20(5.34)$ & $5.00(4.98)$ & $130-131$ \\
\hline CSNtert & 205.6 & $56.30(55.50)$ & $5.70(5.34)$ & $5.00(4.98)$ & $150-152$ \\
\hline CSNisop & 24.3 & $55.30(55.30)$ & $5.20(5.71)$ & $5.00(4.96)$ & $109-110$ \\
\hline
\end{tabular}

Fonte: Dados da pesquisa.

A mudança da banda ${ }^{\circ} \mathrm{C}=\mathrm{O}$ para uma frequência mais baixa está associada à presença do grupo nucleofílico $\mathrm{ClSO}_{2}$ no anel aromático da cumarina, que remove a densidade eletrônica por efeito indutivo(FARAHI; KARAMI; TANURAGHAJ, 2015). O espectro de $\mathrm{RMN}{ }^{1} \mathrm{H}$ da CCIS apresenta um dupleto a 7.9 ppm atribuído ao $\mathrm{H}_{5}$ (Figura 1) com $\mathrm{J}_{\mathrm{H} 5 \mathrm{H} 7}$ a $1.9 \mathrm{~Hz}$, um dupleto a 8.1 ppm $\left(\mathrm{J}_{\mathrm{H} 3 \mathrm{H} 4}\right.$ a $\left.9.76 \mathrm{~Hz}\right)$ atribuído ao $\mathrm{H}_{3}$ do anel pirânico, um duplo dupleto a $7.3\left(\mathrm{~J}_{\mathrm{H} 7 \mathrm{H} 8}\right.$ a $8.50 \mathrm{~Hz}$ e $\mathrm{JH}_{7}-\mathrm{H}_{5}$ a $\left.1,95 \mathrm{~Hz}\right)$, um dupleto a 7.3 ppm $\left(\mathrm{J}_{\mathrm{H} 8 \mathrm{-H} 7}\right.$ a $\left.8.50 \mathrm{~Hz}\right)$ atribuído ao $\mathrm{H}_{8}$ e um dupleto a $6.42 \mathrm{ppm}\left(\mathrm{JH}_{4}-\mathrm{H}_{3}\right.$ de $\left.9,76 \mathrm{~Hz}\right)$ atribuído ao $\mathrm{H}_{4}$. Nenhum sinal foi observado para $\mathrm{H}_{6}$, sugerindo a substituição do grupo na posição 6 do anel aromático (Figura 1).

Figura 1 - Síntese da cumarina-6-clorossulfonada<smiles>O=c1ccc2ccccc2o1</smiles>
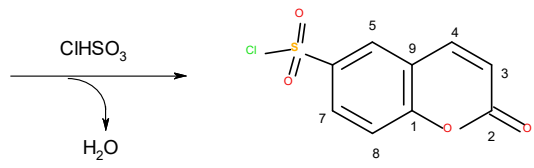

Fonte: Dados da pesquisa.

Os compostos derivados da CCIS foram obtidos a partir da interação do grupo amina com a cumarina-6clorossulfonada através do grupo sulfonato. A solubilidade dos compostos foi testada em diferentes solventes orgânicos. Os compostos exibiram baixa solubilidade em metanol e eram solúveis em clorofórmio e DMSO. Os valores da condutância molar da CSNtert e CSNiso, determinados em solução de dimetilsulfoxido (DMF) à temperatura ambiente, são apresentados no Quadro 1. Os resultados mostram que a CSNtert tem a condutividade maior que $54 \Omega^{-1} \mathrm{~cm}^{2} \mathrm{~mol}^{-1}$, mostrando que é um composto iônico (EL-WAHAB et al., 2004), ou é ionizado em uma solução DMF. No entanto, CSNiso e CSNisop são compostos não iônicos.

Os espectros UV dos compostos foram obtidos em metanol na concentração de $30 \mu \mathrm{M}$ e as respectivas bandas foram mostradas no Quadro 2.

Quadro 2 - Bandas espectroscópicas UV da CClS, CSNtert, CSNiso, CSNisop

\begin{tabular}{|l|c|c|c|c|c|}
\hline Compostos & \multicolumn{5}{|c|}{ Comprimento de onda (nm) } \\
\hline CCIS & 232 & - & 268 & 280 & 308 \\
\hline CSNiso & 232 & - & 268 & 280 & 312 \\
\hline CSNisop & 224 & 236 & 268 & 280 & 312 \\
\hline CSNtert & 224 & 230 & 268 & 280 & 312 \\
\hline
\end{tabular}

Fonte: Dados da pesquisa.

No espectro da sulfonamida foram observadas mudanças na banda de $308 \mathrm{~nm}$ para maior frequência sugerindo interação da amina através do grupo $\mathrm{SO}_{2}$ diminuindo a conjugação da ligação na molécula (Figura 2). As bandas a 268 e $280 \mathrm{~nm}$, associadas com $\pi \rightarrow \pi^{*}$ e $n \rightarrow \pi^{*}$ transições do $C=C$ e $C=O$ bandas do anel aromático, não se alteraram, não mostrando interação da amina via grupo pirânico da cumarina.

Figura 2 - Síntese da CSNiso, CSNisop e CSNtert.

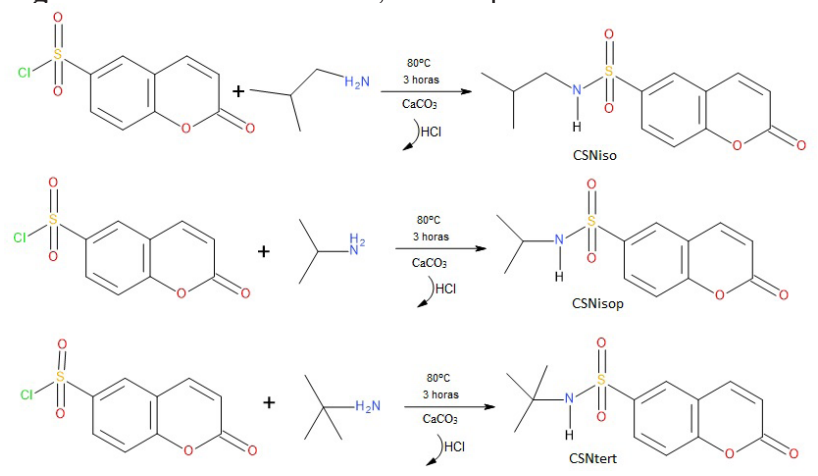

Fonte: Dados da pesquisa.

O espectro no IR de cada composto de sulfonamida CSNiso, CSNtert e CSNisop (Quadro 3) tem uma larga banda na região do $\mathrm{nN}-\mathrm{H}$ mostrando a presença de uma amina secundária em todas as moléculas. Além disso, observou-se a mudança do nCO para maior frequência quando comparado à CClS, indicando alterações na densidade eletrônica nos anéis aromáticos devido à interação do grupo amina como doador eletrônico. A mudança da banda $\mathrm{nS}=\mathrm{O}$ assimétrico e simétrico para uma frequência mais baixa também foram observados, sugerindo a ligação do grupo amina através do átomo $\mathrm{S}$ do grupo sulfônico (FARAHI; KARAMI; TANURAGHAJ, 2015).

Quadro 3 - Bandas IR características $\left(\mathrm{cm}^{-1}\right)$ no espectro da CCIS, CSNtert, CSNiso, CSNisop

\begin{tabular}{|c|c|c|c|c|c|}
\hline Compostos & nN-H & nC=O & nS=O & nC=C & nC-O \\
\hline CCIS & - & 1732 & $1371 ;$ & $1621 ;$ & $1271 ;$ \\
& & & 1166 & 1600 & 1072 \\
\hline CSNiso & 3226 & 1720 & $1369 ;$ & $1622 ;$ & $1267 ;$ \\
& & & 1153 & 1603 & 1067 \\
\hline CSNisop & 3255 & 1728 & $1371 ;$ & $1618 ;$ & $1263 ;$ \\
& & & 1155 & 1603 & 1081 \\
\hline CSNtert & 3277 & 1730 & $1370 ;$ & $1619 ;$ & $1263 ;$ \\
& & & 1147 & 1602 & 1082 \\
\hline
\end{tabular}

Fonte: Dados da pesquisa.

Os espectros de $\mathrm{RMN}$ de ${ }^{1} \mathrm{H}$ e ${ }^{13} \mathrm{C}$ dos novos compostos foram obtidos em DMSO-d 6 e os dados são mostrados nos Quadros 4 e 5. 
O espectro de RMN de ${ }^{1} \mathrm{H}$ da CSNiso, mostra 9 sinais, um a 8,24 ppm atribuído ao hidrogênio $\mathrm{N}-\mathrm{H}$, em campo baixo, quando comparado com o sinal do espectro da isobutilamina a 5,11 ppm. Essa mudança é esperada porque o grupo amina está ligado ao grupo doador de elétrons, deslocando o sinal para campo baixo. Os outros sinais apareceram a 0,82 (d, $6 \mathrm{H}, \mathrm{J} 6,64 \mathrm{~Hz})$ referente ao $\mathrm{H}$ do grupo metil, 1,67 (m, $\left.\mathrm{H}_{2}\right)$ e 2,61 (d, $\left.2 \mathrm{H}_{1}, \mathrm{~J} 1.8 \mathrm{~Hz}\right)$ do grupo isobutil. O sinal do grupo benzênico aparece na região aromática entre 7.98 e 6.64 ppm (Quadro 4).

Quadro 4 - Dados de RMN de ${ }^{1} \mathrm{H}$ da CClS, CSNiso, CSNtert e CSNisop.

\begin{tabular}{|c|c|c|c|c|c|c|c|c|c|c|c|c|}
\hline Compostos & \multicolumn{3}{|c|}{ Carbono - Amina } & \multicolumn{10}{c|}{ Carbono - Cumarina } \\
\hline & C1 & C2 & C3 & C1 & C2 & C3 & C4 & C5 & C6 & C7 & C8 & C9 \\
\hline CCIS & - & - & - & 164,1 & 116,2 & 156,9 & 148,5 & 130,3 & 124,9 & 134,4 & 117,4 & 118,4 \\
\hline CSN iso & 50,1 & 28,1 & 19,9 & 159,4 & 117,6 & 115,5 & 143,7 & 129,7 & 127,3 & 138,8 & 117,5 & 118,9 \\
\hline CSN isop & 45,4 & $23,29\left(2 \mathrm{CH}_{3}\right)$ & - & 159,3 & 117,6 & 155,9 & 143,7 & 129,6 & 127,2 & 137,9 & 117,9 & 118,8 \\
\hline CSN tert & 53,5 & $29,70\left(3 \mathrm{CH}_{3}\right)$ & - & 159,3 & 117,6 & 155,5 & 143,7 & 129,6 & 127,3 & 136,7 & 117,7 & 118,9 \\
\hline
\end{tabular}

Fonte: Dados da pesquisa.

Para o composto CSNtert o ${ }^{13} \mathrm{C}$ NMR mostra 11 sinais. O grupo metil a 29,7 ppm, o carbono quaternário a 53,5 ppm, oito sinais aromáticos do grupo carbonila a 159,3 ppm (Quadro 5).

Quadro 5 - Dados de ${ }^{13} \mathrm{C}$ da CClS, CSNiso, CSNtert e CSNisop.

\begin{tabular}{|c|c|c|c|c|c|c|c|c|c|}
\hline Compostos & \multicolumn{4}{|c|}{ Hidrogênio - Amina } & \multicolumn{3}{c|}{ Hidrogênio - Cumarina } \\
\hline & $\mathbf{H}_{1}$ & $\mathbf{H}_{2}$ & $\mathbf{H}_{3}$ & $\mathbf{N}-\mathbf{H}$ & $\mathbf{H}_{3}$ & $\mathbf{H}_{4}$ & $\mathbf{H}_{5}$ & $\mathbf{H}_{7}$ & $\mathbf{H}_{8}$ \\
\hline CCIS & - & - & - & - & 8.1 & 6.4 & 7.9 & 7.8 & 7.3 \\
\hline \multirow{2}{*}{ CSNiso } & $\begin{array}{c}2.61(\mathrm{~d}, \\
2 \mathrm{H} ; \mathrm{J}= \\
6.8 \mathrm{~Hz})\end{array}$ & $\begin{array}{c}1.67 \\
(\mathrm{~m}, 1 \mathrm{H})\end{array}$ & $\begin{array}{c}0.82(\mathrm{~d}, \\
\mathrm{JH}, \mathrm{CH}_{3},\end{array}$ & 8.2 & 7.9 & 6.6 & 7.9 & 7.7 & 7.6 \\
\hline \multirow{2}{*}{ CSNisop } & $\begin{array}{c}2.51(\mathrm{q}, \\
2 \mathrm{H}, \mathrm{J} \\
=6.5 \mathrm{~Hz})\end{array}$ & $\begin{array}{c}0.97(\mathrm{~d}, \\
6 \mathrm{H}, \mathrm{CH}_{3} \\
\mathrm{~J}=6.5 \mathrm{~Hz})\end{array}$ & - & 8.2 & 8.2 & 6.6 & 7.9 & 7.7 & 7.6 \\
\hline CSNtert & - & $\begin{array}{c}1.13(\mathrm{~s} 6 \mathrm{H}, \\
\left.\mathrm{CH}_{3}\right)\end{array}$ & - & 8.2 & 8.2 & 6.6 & 8.0 & 7.7 & 7.6 \\
\hline
\end{tabular}

Fonte: Dados da pesquisa.

Estas análises sugerem a formação de novas sulfonamidas derivadas da cumarina e com base nos dados espectroscópicos e análises elementares foi possível propor as estruturas moleculares da CSNiso, CSNtert e CSNisop (Figura 2).

$\mathrm{O}$ estudo in vitro da citotoxicidade dos compostos CSNtert, CSNiso e CSNisop com células epiteliais normais (células vero, fibroblasto de rim de macaco) foi realizado para comparar se os novos compostos apresentavam citotoxicidade mais elevada que seus precursores, cumarina e CCIS. Os dados de citotoxicidade estão mostrados no Quadro 6.

Quadro 6 - Estudo citotoxicológico (IC50 mM) da cumarina, CCIS, CSNtert, CSNiso e CSNisop em mM

\begin{tabular}{|c|c|}
\hline Compostos & Vero \\
\hline Cumarina & $0,93 \pm 0,05$ \\
\hline CCIS & $0,76 \pm 0,02$ \\
\hline CSNtert & $1,83 \pm 0,05$ \\
\hline CSNiso & $1,36 \pm 0,03$ \\
\hline CSNisop & $1,24 \pm 0,02$ \\
\hline
\end{tabular}

Fonte: Dados da pesquisa.

Os compostos demonstraram citotoxicidade menor que a cumarina e CCIS contra células normais, sendo CSNtert 2 vezes menos citotóxico que a cumarina e 2,5 vezes menos que a CClS, enquanto que os compostos CSNiso e CSNiso mostraram ser duas vezes menos citotóxicos que a CClS.

\section{Conclusão}

Uma promissora série de cumarinas substituídas com sulfonamida com baixa citotoxicidade e com atividade biológica foram sintetizadas a partir da reação da cumarina6-clorossulfonada com as aminas primárias, tercbutilamina, isobutilamina e isopropilamina, através de uma metodologia rápida e simples com um rendimento expressivo.

\section{Documento de Transparência}

O documento de transparência associado a este artigo pode ser encontrado em uma versão online.

\section{Agradecimentos}

À FAPESP (Processo-2012/14159-9) e à Universidade Anhanguera de São Paulo (UNIAN) por fornecer apoio financeiro e à CAPES para suas bolsas de estudo.

\section{Referências}

BECKER, H.G.O.; BERGER, W. Organikum: organischchemisches Grundpraktikum. Great Britain: Weinheim, 2009.

BRYDA, J. et al. Coumarins as potential supportive medication for the treatment of epilepsy. Acta Neurobiol Exp. v.79, p.126$132,2019$.

CUNHA, M.G. et al. Antimicrobial, anti-adherence and 
antibiofilm activity against Staphylococcus aureus of a 4-phenyl coumarin derivative isolated from Brazilian geopropolis. Microbial Pathogenesis, v.139, p.103855, 2019.

EL-WAHAB, Z.H.A. et al. Co(II), Ce(III) and UO2(VI) BisSalicylatothiosemicarbazide complexes: binary and ternary complexes, thermal studies and antimicrobial activity. Spectrochimica Acta Part A, v.60, p.2861-2873, 2004.

FARAHI, M.; KARAMI, B.; TANURAGHAJ, H.M. Efficient synthesis of a new class of sulfonamide-substituted coumarins. Tetrahedron Letters, v.56, p.1833-1836, 2015.

GÖLCÜ, A. Transition metal complexes of propranolol dithiocarbamate: synthesis, characterization, analytical properties and biological activity. Transit. Met. Chem., v.31, p.405-412, 2006.

GROVER J.; JACHAK S.M. Coumarins as privileged scaffold for anti-inflammatory drug development. RSC $A d v$., v.5, n.49, p.38892-38905, 2015.

JIANG, J. et al. Synthesis and characterization of the ligand based on benzoxazole and its transition metal complexes: DNA-binding and antitumor activity. J. Inorg. Biochem. v.104, n.5, p.583-591, 2010.

KUMAR, A.K. et al. Comprehensive review on coumarins: Molecules of potential chemical and pharmacological in- terest. J. Chem. Pharm. Res., v.7, p.67-81, 2015.

KURT, B.Z., et al. Synthesis of coumarin-sulfonamide derivatives and determination of their cytotoxicity, carbonic anhydrase inhibitory and molecular docking studies. Euro. J. Med. Chem., v.183, p.111702, 2019.

LEE, S.Y. et al. Esculetin suppresses proliferation of human colon cancer cells by directly targeting B-catenin. Cancer Prev. Res., v.6, p.1356-1364, 2013.

LIU, W. et al. Synthesis and in vitro antitumor activity of novel scopoletin derivatives. Bioorg. Med. Chem. Lett., v.22, p.50085012, 2012.
LUNAZZI, L. et al. Stereomutation of axially chiral aryl coumarins. J. Organic Chem., v.75, p.5927-5933, 2010.

NAJMANOVA, I. et al. Cardiovascular effects of coumarins besides their antioxidant activity. Curr. Top. Med. Chem., v.15, p.830-884, 2015. doi: 10.2174/1568026615666150220112437.

NAM, H.; KIM, M.M. Scopoletin has a potential activity for antiaging via autophagy in human lung fibroblasts. Phytomedicine. v.22, p.362-368, 2015. doi: 10.1016/j.phymed.2015.01.004.

NEWMAN, D.J.; CRAGG, G.M. Natural products as sources of new drugs over the 30 years from 1981 to 2010. J. Nat. Prod., v.75, p.311-335, 2012.

NOFAL, Z.M.; EL-ZAHAR, M.I.; ABD EL-KARIM, S.S. Novel coumarin derivatives with expected biological activity. Molecules. v.5, p.99-113, 2000.

PINTO, D.C.G.A.; SILVA, M.A.S. Anticancer Natural Coumarins as Lead Compounds for the Discovery of New Drugs. Curr. Top. Med. Chem., v.17, n.29, p. 3190-3198, 2017. doi: $10.2174 / 1568026618666171215095750$

SABT, A. et al. Novel coumarin-6-sulfonamides as apoptotic anti-proliferative agents: synthesis, in vitro biological evaluation, and QSAR studies. J. Enzyme Inhib Med. Chem., v.33, p.10951107, 2018. doi: 10.1080/14756366.2018.1477137

SINGH, B.K.; RAJOUR, H.K.; PRAKASH, A. Synthesis, characterization and biological activity of transition metal complexes with Schiff bases derived from 2-nitrobenzaldehyde with glycine and methionine. Spectrochim Acta A Mol Biomol Spectrosc. v.94, p.143-151, 2012.

VALDUGA, C.J.; STEFANI H.A.; PETRAGNANI N. Recent advances in selenocyclofunctionalization reactions. Tetrahedron (Oxford. Print). v.57, p.1411-1448, 2001.

ZANI, F.; VICINI, P. Antimicrobial Activity of Some 1,2-Benzisothiazoles Having a Benzenesulfonamide Moiety. Arch. Pharm. Pharm. Med. Chem., v.331, p.219- 223, 1998. 